\title{
Why do ultra-low-frequency MHD oscillations with a discrete spectrum exist in the magnetosphere?
}

\author{
A. S. Leonovich and V. A. Mazur \\ Inst. of Solar-Terrestrial Physics (ISTP), Russian Academy of Science, Siberian Branch, Irkutsk 33, 664 033, Russia \\ Received: 19 August 2004 - Revised: 18 January 2005 - Accepted: 21 January 2005 - Published: 30 March 2005
}

\begin{abstract}
A new concept of the global magnetospheric resonator is suggested for fast magnetosonic waves in which the role of the resonator is played by the near-Earth part of the plasma sheet. It is shown that the magnetosonic wave is confined in this region of the magnetosphere within its boundaries. The representative value of the resonator's eigenfrequency estimated at $f \sim 1 \mathrm{MHz}$ is in good agreement with observational data of ultra-low-frequency MHD oscillations of the magnetosphere with a discrete spectrum $(f \sim 0.8,1.3$, $1.9,2.6 \ldots \mathrm{MHz}$ ). The theory explains the ground-based localization of the oscillations observed in the midnight-morning sector of the high-latitude magnetosphere.
\end{abstract}

Keywords. Magnetospheric physics (Magnetotail; MHD waves and instabilities; plasma sheet)

\section{Introduction}

The Earth's magnetosphere represents a low-density plasma cavity within the solar wind stream separated from it by a sharp boundary, the magnetopause. For quite some time has been hypothesized that this cavity would serve as a resonator for fast magnetosonic waves (McClay, 1970; Southwood and Kivelson, 1986; Kivelson and Southwood, 1986). The resonator's eigenmodes (cavity modes, global modes) can be driven by instabilities at the magnetopause (McKenzie, 1970) or by unsteady processes in the solar wind (Southwood and Kivelson, 1990). Such oscillations, which are the largestscale and lowest-frequency MHD oscillations of the magnetosphere, must have a discrete spectrum. Through the field line resonance mechanism, the magnetosonic eigenmodes can excite standing Alfvén waves on those field lines, the local Alfvén frequency of which coincides with the resonator's eigenfrequency (Southwood and Kivelson, 1986; Leonovich and Mazur, 1989). However, such oscillation, due to its large

Correspondence to: A. S. Leonovich

(leon@iszf.irk.ru) scale, can be observed directly on the Earth's surface without the intermediate excitation of the standing Alfvén wave.

A reasonable amount of research has been done to date, addressing the theory of global MHD eigen-oscillations of the magnetosphere (in addition to the references cited above, see also Zhu and Kivelson, 1989; Lee and Lysak, 1991; Leonovich and Mazur, 2001). Nevertheless, the status of the theory cannot be recognized as satisfactory. The Earth's magnetosphere is not a closed cavity. The geotail extends into the solar wind to an indefinitely large distance. Therefore, magnetosonic waves are thought of as being able to freely escape along it. In such a case, however, it is beyond reason to hope for a closed resonator with a discrete spectrum of frequencies. This difficulty of the theory has not yet received any acceptable resolution.

In the meantime, oscillations, having the basic properties of global MHD modes, were detected. They are observed both with HF radars (Ruohoniemi et al., 1991; Samson and Harrold, 1992) and at ground-based magnetometer networks (Wanliss et al., 2002). These oscillations have clearly pronounced maxima in the oscillation spectrum at the frequencies $f \sim 1.3,1.9,2,6$, and $1.4 \mathrm{MHz}$. Sometimes oscillations with a frequency $0.8 \mathrm{MHz}$ (Lessard et al., 2003) are observed. The oscillations are normally recorded in the midnight-morning sector at latitudes from $60^{\circ}$ to $80^{\circ}$, with a low disturbance level of the magnetosphere $K_{p} \sim 1-3$ (Harrold and Samson, 1992; Samson and Harrold, 1992). The frequencies of such oscillations are independent of the latitude of the observation point and remain virtually unchanged both from event to event and within a single observing interval.

The experimental detection of ultra-low-frequency hydromagnetic oscillations with a discrete spectrum made the problem of constructing an adequate theory still more urgent. Unquestionably, such a theory must include the presence of the geotail. Some authors (Harrold and Samson, 1992; Samson and Harrold, 1992; Samson and Rankin, 1994) made an attempt to treat the observed oscillations as the eigenmodes of the waveguide in the geotail rather than of the resonator. However, the eigenmodes of the waveguide produce 


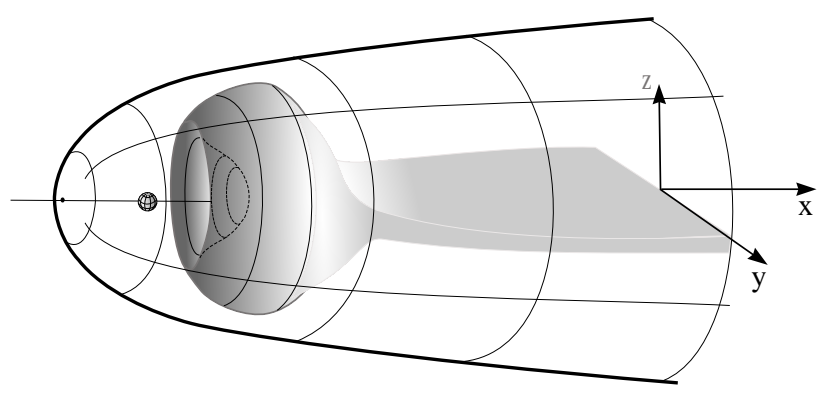

Fig. 1. Model of the magnetosphere. The plasma sheet appears gray. The figure does not show the asymmetry of the near-Earth plasma sheet about the midday-midnight meridian. The outer boundary of the figure is the magnetopause separating the magnetosphere and the magnetosheath.

no discrete spectrum, since the wavelength along the waveguide can be an arbitrary one. The authors of the cited papers recognize that this difficulty has no satisfactory resolution in terms of the theory they suggested (Samson and Rankin, 1994). Furthermore, there is a number of other problems inherent in this theory. So, inside this concept it is difficult to explain such low frequencies of the oscillations, and also why they do not disappear into the geotail along the waveguide. In this paper we suggest a new concept of the resonator for ultra-low-frequency magnetosonic waves in which it is possible to overcome most of the difficulties inherent in previous work.

\section{The resonator in the near-Earth part of the plasma sheet}

The basic idea of this study implies that the role of the resonator is played by the near-Earth part of the plasma sheet (NEPS). This important large-scale element of the magnetosphere represents a lentil-shaped region (see Fig. 1), filled with a sufficiently dense plasma $\left(n \sim 1 \mathrm{~cm}^{-1}\right)$. Combined with a reasonably low value of the magnetic field $(B \sim 5-10 \mathrm{nT})$, this leads to minimum (for the entire magnetosphere) representative values of the Alfvén velocity $A=B / \sqrt{4 \pi m_{p} n}$ $\sim 10^{2} \mathrm{~km} / \mathrm{s}$. It is not inconceivable that the values $A \sim(30$ 50) $\mathrm{km} / \mathrm{s}$ exist (Borovsky et al., 1998). The boundary of the plasma sheet presented in Fig. 1 should be understood as the surface, which divides the magnetosphere cavity into the areas with the big and small values of Alfvén velocity. Therefore it does not reach the Earth on approach, to which the values of a geomagnetic field and Alfvén velocity are growing.

The velocity of fast magnetosonic waves is represented by the following expression, $C_{f}=\sqrt{A^{2}+S^{2}}$, where $S=\sqrt{\gamma P / \rho}$ - sound velocity in plasmas. In contrast to Alfvén velocity, the distribution of sound velocity in the magnetosphere is much less known. This is due to the fact that sound velocity is a function of temperature, whose distribution in the magnetosphere is determined much worse than the distribution of the magnetic field and density of plasma. So, in the neutral sheet (and in the equatorial part of the NEPS), the temperature of plasma is great enough for value $\beta=B^{2} / 8 \pi n T \approx A^{2} / S^{2} \gg 1$ (Borovsky et al., 1998). This means that gas-kinetic pressure of plasma is here much greater than the magnetic pressure. The plasma sheet is the hottest area of the magnetosphere. Its steady state is supported by a sharp gradient of magnetic pressure in the direction from the neutral sheet to the tail lobes.

The conditions in the NEPS are different completely. The geomagnetic field here is a dipole-like one. The dipole field is force-free, in spite of the fact that its field lines are curvelinear. Therefore, it is possible to suppose that the geomagnetic field in the NEPS area is almost a force-free one. This means that the plasma pressure does not play an essential role in the forces balance which provides equilibrium of the magnetic configuration. We will consider the magnetosphere in an undisturbed quiet state. It is possible to suppose that in such a steady state the volume-average of a flux tube condition $\beta \lesssim 1$ is held in the NEPS, i.e. on the order of magnitude $C_{f} \approx A$. The insufficient difference of the eigenfrequencies' estimation carried out below, associated with considering a sound velocity, does not influence the order of value in the final result. In the tail lobes, where $\beta \ll 1$, the influence of sound velocity can be entirely neglected.

The NEPS continually changes to a flat neutral sheet, separating the magnetotail into two lobes. These parts of the plasma sheet form a unit plasma configuration. Nevertheless, treating them separately is quite justified, both in terms of the morphology and from the character of propagation of magnetosonic waves.

The characteristic radius of the NEPS roughly coincides with the cross section of the geotail in this part of the magnetosphere $a \sim 10^{5} \mathrm{~km}$. The inner edge of the plasma sheet lies at (5-10) $R_{E}$ from the Earth, and the outer edge of the NEPS is at the distance $(20-30) R_{E}$ in the antisunward direction. Thus, the characteristic size of the NEPS in this direction $\Delta$ is of the same order of magnitude $\Delta \sim 10^{5} \mathrm{~km}$. The above values of the Alfvén velocity $A$ and of the size of the region $a \sim \Delta$ lead to the characteristic value of the frequency of the possible eigenmode $f \sim A / a \sim 1 \mathrm{MHz}$, in full agreement with observed values.

Of course, before speaking about the eigenmode frequency, one has to make sure that the fast magnetosonic wave can be confined to within the NEPS. In the radial direction, the confinement is caused by the presence of a sharp boundary, the magnetopause, at which the Alfvén velocity changes abruptly from the values $(300-500) \mathrm{km} / \mathrm{s}$ in the magnetosphere to substantially lower values $(50-100) \mathrm{km} / \mathrm{s}$ in the magnetosheath. On such a boundary there is taking place partial reflection of the magnetosonic wave which increases with the increasing Alfvén velocity difference. The transition coefficient $\kappa_{t}\left(\kappa_{t}=1-\kappa_{r}\right.$, where $\kappa_{r}$ is the reflection coefficient) is equal, on the order of magnitude, to the ratio of the Alfvén velocities on the outer and inner sides of the magnetopause - for the aforementioned values $\kappa_{t} \sim 0.1-0.3$. Thus, at this boundary there is a sufficiently effective confinement 
of magnetosonic waves taking place. Partial penetration of the wave through the magnetopause leads to the resonator's $Q$ factor $Q \sim 3-10$.

Before proceeding to the discussion of the reason why the wave is reflected by the outer and inner edges of the plasma sheet, we present some simple evidence from the theory of MHD resonators. In a homogeneous cold plasma residing in a homogeneous magnetic field, the frequency of a fast magnetosonic wave is given by the relation

$\omega^{2}=\left(k_{x}^{2}+k_{y}^{2}+k_{z}^{2}\right) A^{2}$,

where $k_{x}, k_{y}, k_{z}$ stand for the wave vector components. If the wave is confined (say, by reflecting walls) in a square box with the size along its axes $a_{x}, a_{y}, a_{z}$, then the wave vector components are quantized

$k_{x}=\frac{\pi n_{x}}{a_{x}}, \quad k_{y}=\frac{\pi n_{y}}{a_{y}}, \quad k_{z}=\frac{\pi n_{z}}{a_{z}}$,

where the wave numbers $n_{x}, n_{y}, n_{z}$ can take a natural series of values. If the medium within the resonator is inhomogeneous in one coordinate, say, $x$, and if the inhomogeneity is such that the WKB approximation is applicable, then Eq. (1) can be treated as the equation defining a quasi-classical wave vector $k_{x}=k_{x}(x, \omega)$ :

$k_{x}^{2}=\frac{\omega^{2}}{A^{2}(x)}-k_{y}^{2}-k_{z}^{2}$,

in which case the relations Eq. (2) hold for $k_{y}$ and $k_{z}$ as before. The turning point (more exactly, surface) at which $k_{x}^{2}(x)=0$ separates the opaque region, where $k_{x}^{2}(x)<0$, from the transparent region, where $k_{x}^{2}(x)>0$. It is easy to see that in the opaque region the Alfvén velocity has larger values compared to the transparent region. The turning surface can be treated as a reflection surface where the wave is reflected from the opaque region. The reflection coefficient $\kappa_{r}$ increases with the increasing width of the opaque region. If the width of the opaque region is much larger than the characteristic wavelength, then $\kappa_{r} \approx 1$. If the transparent region is surrounded by turning points (surfaces) on both sides, then it is possible for a resonator to be produced. The frequency spectrum of such a resonator within the WKB approximation is determined by the Bohr-Sommerfeld quantization condition.

In the three-dimensional inhomogeneous case, these notions all retain their meaning. The turning surface, which is not a plane in the general case, separates the transparent region, with a relatively small value of the Alfvén velocity, from the opaque region where the values of the Alfvén velocity are larger. We now return to the discussion of the NEPS. The confinement of the magnetosonic wave within this region in the earthward direction is caused by the presence of the turning surface in this direction. The existence of this surface is caused by a significant increase in the Alfvén velocity from $A \sim 10^{2} \mathrm{~km} / \mathrm{s}$ in the NEPS to $A \sim(2-3) \cdot 10^{3} \mathrm{~km} / \mathrm{s}$ in the near-Earth region. This turning surface virtually coincides with the inner edge of the plasma sheet, where an abrupt change in the Alfvén velocity occurs. The region between the inner edge of the plasma sheet and the Earth is an opaque region for the wave under consideration.

The outer edge of the NEPS is a reflection surface for the same reason. Behind it there are the tail lobes in which the Alfvén velocity reaches even larger values than in the nearEarth region $A \sim(3-10) \cdot 10^{3} \mathrm{~km} / \mathrm{s}$. The only question remains with regards to the cleft-shaped region of transition from the NEPS to the neutral sheet. This region may be treated as a peculiar kind of hole in the outer edge of the NEPS on which the neutral plasma sheet "abuts". It would appear that the magnetosonic wave can freely escape to it. For the oscillations under consideration, this is not the case, however.

The neutral plasma sheet may be regarded as a planar waveguide (Siskoe, 1969). Let the axis $x$ be directed along the waveguide axis in the antisunward direction, and axis $y$ azimuthally, and the axis $z$ across the neutral sheet. Equations (2) and (3) can be applied for such a waveguide. On the order of magnitude $a_{y} \sim a \sim 10^{5} \mathrm{~km}$, and $a_{z} \sim 10^{4} \mathrm{~km}$ is the thickness of the neutral sheet. For the fundamental mode of oscillations in the such waveguide $k_{y} \sim \pi / a, k_{z} \sim \pi / a_{z}$, i.e. $k_{y}^{2} \ll k_{z}^{2}$. The frequency of the oscillation (eigenmode) under consideration, as has already been pointed out above, is $\omega \sim \pi A / a$, where $A$ is the characteristic Alfvén velocity in the NEPS. The velocity of fast magnetosonic waves in the neutral sheet is greater than its average value in the NEPS due to a higher temperature. Then within the neutral sheet we have

$$
\frac{\omega^{2}}{C_{f}^{2}} \ll k_{z}^{2},
$$

and, according to Eqs. (2) and (3)

$k_{x}^{2}=-k_{z}^{2} \sim-\frac{\pi^{2}}{a_{z}^{2}}$.

The resonator's fundamental eigenmode cannot propagate in the neutral sheet but penetrates to its depth only by about its thickness $a_{z}$. In other words, the turning surface, from which full reflection of magnetosonic waves under consideration occurs, is formed in the NEPS in the antisunward direction on the whole geomagnetic tail section, including the plasma sheet. Thus, the fast magnetosonic wave under consideration is confined on all the boundaries surrounding the NEPS.

\section{Conclusion}

We now formulate the results obtained in this study and the implications that follow directly from them.

1. The near part of the plasma sheet (NEPS) can play the role of the resonator for fast magnetosonic waves. These waves are reflected both by the resonator's side boundary, which form part of the magnetopause, and by the inner and outer edges of the NEPS. Because of the 
small thickness of the neutral sheet, modes of the resonator cannot penetrate into its depth.

2. The characteristic value of the frequency of the resonator's fundamental modes is $\sim 1 \mathrm{MHz}$, which is in good agreement with observed frequencies of ultra-lowfrequency oscillations with a discrete spectrum of frequencies. A complete calculation of the spectrum of the resonator's eigen-oscillations requires a definite model for the NEPS, and this will be the subject of our separate study. It is clear that by fitting the model parameters, it is always possible to achieve a good reconciliation with observations of one or two eigenfrequencies. It is of interest, however, to what extent the properties of the spectrum of such a resonator can be reproduced in general.

3. The $Q$ factor of the resonator discussed here is not very high. The loss of energy caused by the escape of the mode through the magnetopause gives the values $Q \sim 3-10$. If the other loss mechanisms are important, then the $Q$ factor will be still smaller. But this value of the $Q$ factor is large enough for the resonator driven by a sudden impulse (a shock wave from the solar wind, say) to show a train of oscillations of $3-10$ periods.

4. Standing Alfvén modes rather than the cavity modes themselves (the near-Earth part of the magnetosphere is the opacity region for them) are observed on the ground. They are driven by the field line resonance mechanism along field lines passing through the resonator's region. The fact that the resonator is represented by the NEPS explains in a natural way the ground-based localization of observed ultra-low-frequency oscillations with a discrete spectrum, both in latitude $60^{\circ}-80^{\circ}$ and in longitude - in the midnight-morning sector. The displacement of the oscillations relative to their symmetric midnight position can be explained by the convection of magnetospheric plasma that makes the NEPS move into the morning sector (Elphic et al., 1999).

5. Unfortunately, the concept suggested in this study cannot to explain the surprising stability of the frequencies observed. Incidentally, this difficulty is inherent in any version of the theory of magnetospheric resonator. The magnetosphere is not stable, and its parameters vary over a rather wide range, including the parameters of the NEPS. One conceivable explanation for this can be found through the following reasoning. The magnetospheric cavity modes are long-period oscillations $\left(T \sim 10^{3} \mathrm{~s}\right)$. In order for them to stand out against the dynamics of the magnetosphere itself, this must be relatively stable on time intervals on the order of $1 \mathrm{~h}$ or longer. Indeed, oscillations are most frequently observed at the level of a disturbance corresponding to $\mathrm{K}_{p} \sim 1-3$. In this case the magnetospheric parameters lie within a much narrower range compared to their extreme values. In other words, possibilities for the existence and excitation of cavity modes are only created within a relatively narrow range of possible states of the magnetosphere, including the NEPS. Besides, it can be suggested that such an integral characteristic of the resonator as its eigenfrequency (on the order of magnitude, it is $f \sim A / a$ ) is more stable than separately the parameters $A$ and $a$ characterizing it.

Acknowledgements. We are grateful to Mikhalkovsky, V. G. for his assistance in preparing the English version of the manuscript.

This work was partially supported by the Russian Foundation for Basic Research, grants RFBR-No:04-05-64321 and RFBR-GFENNo:02-05-39020.

Topical Editor T. Pulkkinen thanks a referee for her/his help in evaluating this paper.

\section{References}

Borovsky, J. E., Thomsen, M. F., Elphic, R. C., Cayton, T. E., and McComac, D. J.: The transport of plasma sheet material from the distant tail to geosynchronous orbit, J. Geophys. Res., 103, 20 297-20331, 1998.

Elphic, R. C., Thomsen, M. F., Borovsky, J. E., and McComas, D. J.: Inner edge of the electron plasma sheet: empirical models of boundary location, J. Geophys. Res., 104, 22 679-22 693, 1999.

Harrold, B. G. and Samson, J. C.: Standing ULF modes of the magnetosphere: a theory, Geophys. Res. Let., 19, 1811-1814, 1992.

Kivelson, M. G. and Southwood, D. J.: Coupling of global magnetospheric MHD eigenmodes to field line resonances, J. Geophys. Res., 91, 4345-4351, 1986.

Lee, D.-H. and Lysak, R. L.: Monochromatic ULF wave excitation coupling in the dipole magnetosphere, J. Geophys. Res., 96, 5811-5823, 1991.

Leonovich, A. S. and Mazur, V. A.: Resonance excitation of standing Alfvén waves in an axisymmetric magnetosphere (monochromatic oscillations), Planet. Space Sci., 37, 10951108, 1989.

Leonovich, A. S. and Mazur, V. A.: On the spectrum of magnetosonic eigenoscillations of an axisymmetric magnetosphere, J. Geophys. Res., 106, 3919-3928, 2001.

Lessard, M. R., Hanna, J., Donovan, E. F., and Reeves, G. D.: Evidence for a discrete spectrum of persistent magnetospheric fluctuations below $1 \mathrm{Mhz}$, J. Geophys. Res., 108(A3), 1125, doi:10.1029/2002JA009311, 2003.

McKenzie, J. F.: Hydromagnetic wave interaction with the magnetopause and the bow shock, Planet. Space Sci., 18, 1-23, 1970.

McClay, J. F.: On resonant modes of a cavity and the dynamical properties of micropulsations, Planet. Space Sci., 18, 1673-1682, 1970.

Ruohoniemi, J. M., Greenwald, R. A., and Baker K. B.: HF radar observations of Pc5 field line resonances in the midnight/early morning MLT sector, J. Geophys. Res., 96, 15 697-15 710, 1991.

Samson, J. C. and Harrold, B. G.: Field line resonances associated with waveguides in the magnetosphere, Geophys. Res. Lett., 19, 441-444, 1992.

Samson, J. C. and Rankin, R.: The coupling of solar wind energy to MHD cavity modes, waveguide modes and field line resonances in the Earth's magnetosphere, in: Solar Wind Sources of Magnetospheric Ultra-Low-Frequency Waves, Geophys. Monogr. Ser., vol. 81, edited by: Engebretson, M. J., Takahashi, K. and Scholer, M., AGU, Washington, D.C., 253-264, 1994. 
Siskoe, G. L.: Resonant compressional waves in the geomagnetic tail, J. Geophys. Res., 74, 6482-6486, 1969.

Southwood, D. J. and Kivelson, M. G.: The effect of parallel inhomogeneity of magnetospheric hydromagnetic wave coupling, J. Geophys. Res., 91, 6871-6877, 1986.

Southwood, D. J. and Kivelson, M. J.: The magnetohydrodynamic response of magnetospheric cavity to changes in solar wind pressure, J. Geophys. Res., 95, 2301-2310, 1990.
Wanliss, J. A., Rankin, R., and Samson, J. C.: Field line resonances in a stretched magnetotail: CANOPUS optical and magnetometer observations, J. Geophys. Res., 107, SMP9:1-12, 2002.

Zhu, X. and Kivelson, M. G.: Global mode ULF pulsations in a magnetosphere with a nonmonotonic Alfvén velocity profile, J. Geophys. Res., 94, 1479-1485, 1989. 